\title{
VS-FCM: Validity-guided Spatial Fuzzy $c$-Means Clustering for Image Segmentation
}

\author{
Bo-Yeong Kang ${ }^{1}$ and Dae-Won Kim ${ }^{2}$ \\ 1 School of Mechanical Engineering, Kyungpook National University, Daegu 702-701, Korea \\ 2 School of Computer Science and Engineering, Chung-Ang University, Seoul 156-756, Korea
}

\begin{abstract}
In this paper a new fuzzy clustering approach to the color clustering problem has been proposed. To deal with the limitations of the traditional FCM algorithm, we propose a spatial homogeneity-based FCM algorithm. Moreover, the cluster validity index is employed to automatically determine the number of clusters for a given image. We refer to this method as VS-FCM algorithm. The effectiveness of the proposed method is demonstrated through various clustering examples.
\end{abstract}

Key Words : Fuzzy clustering, Color clustering, Fuzzy c-means

\section{Introduction}

The objective of color clustering is to divide a color set into $c$ homogeneous color clusters, which is used in color image segmentation and recognition. Color clustering is an inherently ambiguous task because color boundaries between objects are often blurred and distorted due to the image acquisition process [1]. Since Bezdek first reported his fuzzy set theoretic image segmentation algorithm for aerial images [1], numerous reports have appeared detailing the superior manner in which the fuzzy $c$-means (FCM) algorithm handles uncertainties $[1,2,3,4,5,6,7]$; FCM is less prone to falling into local optima than crisp clustering algorithms.

In spite of the advantages of the fuzzy clustering approach, the traditional FCM-type methods have a number of limitations:

Limit 1. The initialization step is important because different selections of the initial cluster centroids can potentially lead to different local optima or different partitions. However, there is no general agreement regarding which initialization scheme gives the best results $[1,3]$. Most algorithms developed to date assign the random locations to the initial centroids because such a selection will definitely converge in the iterative process.

Limit 2. Traditional spatial FCM algorithms are limited in formulating the influence of the spatial neighborhood

Manuscript received Sep. 14, 2009; revised Oct. 30, 2009.

Corresponding Author:dwkim@cau.ac.kr (Dae-Won Kim)

This work was supported by Basic Science Research Program through

the National Research Foundation of Korea(NRF) grant funded by the

Korea government(MEST)(No.2009-0064861) of a data point on calculating its degree of membership to a given cluster. As Liew pointed out [6], in the original spatial FCM algorithm put forwarded by Tolias and Panas [5], the influence of neighboring color points on a central color point is binary in that it is implemented through the addition and subtraction of a fixed constant. The adaptive spatial FCM algorithm of Liew still does not provide an explicit mathematical formulation for handling homogeneous regions.

Limit 3. The FCM requires the user to pre-define the number of clusters; however, it is not always possible to know the number of clusters in advance.

In this study, we present a new fuzzy approach to the color clustering problem that effectively handles the aforementioned limitations.

\section{The Proposed Method}

\subsection{Initial Centroids Selection}

To solve the initialization problem, we exploit the work of Kim et al. [8] for selecting initial centroids of the FCM. We briefly describe the method as follows.

The key concept of the method is that the dominant and vivid data in a given data set are unlikely to belong to the 
same cluster; these distinguishable dominant data are then used to guess the initial cluster centroids. To obtain the dominant colors from a color set $x \in X$, the following two factors should be considered; (1) a set of reference data and (2) a measure of similarity between a data point and the reference color. Let us suppose that a set of reference data containing all the major features in a given data set can be compiled. For example, when applied to the image segmentation, the reference data might be composed of a set of distinguishable colors such as red, green, and blue with which to measure similarity between color data.

Secondly, the similarity between a color data and a reference color is calculated by the degree of membership of each color point to a reference color. Suppose a color point, denoted by $x=\left(x_{L}, x_{a}, x_{b}\right) \in X$, is a point in the CIELAB color space. Likewise, the $i$-th reference color, denoted by $r_{i}=\left(r_{L}^{i}, r_{a}^{i}, r_{b}^{i}\right) \in R$, is a point in the CIELAB space. The distance between $x$ and $r_{i}$, denoted by $\delta\left(x, r_{i}\right)$, is calculated from the CIELAB color difference formula [9]:

$$
\delta\left(x, r_{i}\right)=\sqrt{\left(x_{L}-r_{L}^{i}\right)^{2}+\left(x_{a}-r_{a}^{i}\right)^{2}+\left(x_{b}-r_{b}^{i}\right)^{2}}
$$

Then, the degree of membership $\mu_{r_{i}}(x)$ of $x$ to the reference color $r_{i}$ is defined as:

$$
\mu_{r_{i}}(x)=\left(\sum_{j=1}^{k} \frac{\delta\left(x, r_{i}\right)}{\delta\left(x, r_{j}\right)}\right)^{-1}
$$

where $r_{i} \neq r_{j}, r_{j} \in R$. If $x$ exactly coincides with $r_{i}$, it has $\mu_{r_{i}}(x)=1.0$. Conversely, if $x$ exactly coincides with another reference color $r_{j}$, then $\mu_{r_{i}}(x)=0.0$. When $x$ does not exactly coincide with any reference colors, the membership value is determined using Eq. 2 .

To determine the dominant colors, we compute the membership degrees between all $x_{j} \in X$ and $r_{i} \in R$. Each $r_{i}$ has two additional attributes, denoted $\mu_{i}$ and $p_{i} . \mu_{i}$ indicates the highest membership degree obtained by computing $\mu_{r_{i}}\left(x_{j}\right)$ for all $x_{j} \in X$, and $p_{i}$ indicates the closest color point $x_{j}$ to $r_{i}$. For $x_{j} \in X$, we compute the membership degree for each of the reference colors, and update $\mu_{i}$ and $p_{i}$. When the computation is completed, the reference colors are sorted by $\mu_{i}$ in decreasing order, which is represented by $R^{s}=\left(r_{s 1}, r_{s 2}, \ldots, r_{s k}\right)$ where $r_{s 1}$ has the highest value of $\mu_{i}$ and $r_{s k}$ has the lowest one.

Given $R, R^{s}$, and the number of clusters $c$, the set of dominant colors is defined as $D=\left\{d_{i} \mid d_{i}=r_{s i}, 1 \leq\right.$ $i \leq c\}$. Thus $D$ consists of the first $c$ reference colors in the sorted list. Having established the dominant colors, the initial cluster centroids $\left(V_{0}=\left\{v_{1}, v_{2}, \ldots, v_{c}\right\}\right)$ are assigned to the color point $x_{i}\left(=p_{i}\right)$ that is closest to the dominant color $d_{i}$. Formally given input $X=\left\{x_{1}, \ldots, x_{n}\right\}$, $R=\left\{r_{1}, \ldots, r_{k}\right\}$, and $c$, the initialization step is described as follows:
S1: Compute the membership degree $\mu_{r_{i}}\left(x_{j}\right)$ for all $x_{j} \in X$

S2: Update $\mu_{i}$ and $p_{i}$ for all $r_{i} \in R$

S3: Sort $r_{i} \in R$ in descending order of $\mu_{i}$ to obtain $R^{s}$

S4: Determine $d_{i}$ from $R^{s}$ and assign $p_{i} \in d_{i}$ to $v_{i}$

\subsection{S-FCM: Spatial Homogeneity FCM}

To exploit the spatial clustering, we employ the notion of homogeneity extracted from spatial domain. Homogeneity is a local information that reflects the uniformity of a region [10]; when a region is homogeneous, data in the homogeneous region are likely to have similar characteristics. For each color point, the homogeneity value for each color point is computed in terms of two components, variance and discontinuity in the CIELAB space. In this scheme, the higher the homogeneity value of color $x_{j} \in X$, the greater the likelihood that $x_{j}$ and its spatial neighborhood belong to the same region.

The variance is a measure of the differences of color values in a predefined spatial neighborhood. Let $x_{j}$ be a color point in the CIELAB space, and let $S_{j}$ denote a spatial neighborhood in two-dimensional space, specified to be a $d \times d$ window size centered at $x_{j}$. Then, the variance $\sigma^{2}\left(x_{j}\right)$ of color $x_{j}$ is defined as:

$$
\sigma^{2}\left(x_{j}\right)=\frac{1}{d^{2}} \sum_{x_{k} \in S_{j}}\left(x_{k}-m_{j}\right)^{2}
$$

where

$$
m_{j}=\frac{1}{d^{2}} \sum_{x_{k} \in S_{j}} x_{k}
$$

$m_{j}$ is the mean value of the color data in $S_{j}$.

The discontinuity $g\left(x_{j}\right)$ of color $x_{j}$ is obtained through the first derivatives at spatial coordinate $x_{j}$, which is defined as the magnitude of the gradient between $x_{j}$ and $S_{j}$ [10]. When the data to be clustered are given in two dimensional space, the discontinuity means the first derivative gradients for the horizontal $\left(G_{x_{j}, h}^{2}\right)$ and vertical $\left(G_{x_{j}, v}^{2}\right)$ directions at $x_{j}$ :

$$
\begin{aligned}
g\left(x_{j}\right) & =\left[G_{x_{j}, h}^{2}+G_{x_{j}, v}^{2}\right]^{1 / 2} \\
& =\left[\left(\frac{\partial g}{\partial x_{j, h}}\right)^{2}+\left(\frac{\partial g}{\partial x_{j, v}}\right)^{2}\right]^{1 / 2}
\end{aligned}
$$

The two measures, $\sigma^{2}\left(x_{j}\right)$ and $g\left(x_{j}\right)$, have different scales that needs to be reconciled through a normalization by their maximum values. These normalized values of the variance, $N\left(\sigma^{2}\left(x_{j}\right)\right)$, and discontinuity, $N\left(g\left(x_{j}\right)\right)$, are now used to define the homogeneity value $h\left(x_{j}\right)$ of data $x_{j}$ :

$$
h\left(x_{j}\right)=1-N\left(\sigma^{2}\left(x_{j}\right)\right) \times N\left(g\left(x_{j}\right)\right)
$$


where $0 \leq h\left(x_{j}\right) \leq 1$

The goal of the present study is to cluster a set of color data $X=\left\{x_{1}, x_{2}, \ldots, x_{n}\right\}$ into $c$ homogeneous groups. To achieve this, we propose a spatial homogeneity-based fuzzy $c$-means (S-FCM) algorithm. The objective of the S-FCM algorithm is to cluster the data $X$ into $c$ clusters $\left(F_{1}, F_{2}, . ., F_{c}\right)$ by minimizing $J_{m}$ :

$$
J_{m}(U, V: X)=\sum_{i=1}^{c} \sum_{j=1}^{n}\left(\mu_{F_{i}}\left(x_{j}\right)\right)^{m}\left\|x_{j}-v_{i}\right\|^{2}
$$

where

$$
\mu_{F_{i}}\left(x_{j}\right)=\frac{1}{n_{j}}\left(\mu_{i j}+\sum_{x_{k} \in S_{j}} \mu_{i k} h\left(x_{k}\right)\right)
$$

and

$$
\mu_{i j}=\left(\sum_{z=1}^{c}\left(\frac{\left\|x_{j}-v_{i}\right\|^{2}}{\left\|x_{j}-v_{z}\right\|^{2}}\right)^{\frac{1}{m-1}}\right)^{-1}
$$

subject to $0 \leq \mu_{F_{i}}\left(x_{j}\right) \leq 1, \sum_{i=1}^{c} \mu_{F_{i}}\left(x_{j}\right)=1$, and $0<\sum_{j=1}^{n} \mu_{F_{i}}\left(x_{j}\right)<n$. In Eq. $9, \mu_{i j}$ is obtained by computing relative distances between clusters in a color feature space. $x_{k} \in S_{j}$ is a spatial neighborhood of $x_{j}$, defined as a $d \times d$ window. $n_{j}\left(=d^{2}\right)$ is the number of $x_{j}$ and its neighboring data, and $h\left(x_{k}\right)$ is the homogeneity value of color $x_{k}$.

We can see that $\mu_{F_{i}}\left(x_{j}\right)$ is influenced by the relations $\left(\mu_{i k}\right)$ between $x_{k} \in S_{j}$ and $F_{i}$ and, furthermore, the extent of this influence is controlled by the degree of homogeneity $h\left(x_{k}\right)$. When $x_{j}$ is located in a homogeneous region, it is useful to exploit the relations between the neighborhood and the centroid because data in a homogeneous region are likely to belong to the same cluster. In contrast, when $x_{k} \in S_{j}$ lies in a nonhomogeneous region such as an edge, the influence of $x_{k}$ on $x_{j}$ should be made as small as possible. Thus, the degree to which $x_{k}$ influences $x_{j}$ is determined through $h\left(x_{k}\right)$. Formally given input $X=\left\{x_{1}, \ldots, x_{n}\right\}, R=\left\{r_{1}, \ldots, r_{k}\right\}, c, m, \epsilon$, and $d$, the $\mathrm{S}-\mathrm{FCM}$ procedural steps are described as follows:

S1: $J(0) \leftarrow \infty, t \leftarrow 1$, run the initialization procedure

S2: Compute $h\left(x_{j}\right)$ and $\mu_{F_{1}}\left(x_{j}\right)(t), \ldots, \mu_{F_{c}}\left(x_{j}\right)(t)$

S3: Compute the objective function $J(t)$

S4: while $|J(t)-J(t-1)|>\epsilon$ do (S5, S6, S7, S8)

S5: $t \leftarrow t+1$

S6: $v_{i}(t) \leftarrow \sum_{j=1}^{n}\left(\mu_{F_{i}}\left(x_{j}\right)(t)\right)^{m} x_{j} / \sum_{j=1}^{n}\left(\mu_{F_{i}}\left(x_{j}\right)(t)\right)^{m}$

S7: Update $\mu_{F_{1}}\left(x_{j}\right)(t), \ldots, \mu_{F_{c}}\left(x_{j}\right)(t)$ for all $x_{j} \in X$

S8: Compute $J(t)$ and go to S4

\subsection{VS-FCM: Validity-Guided SFCM}

Most of clustering methods including the S-FCM algorithm require the user to pre-define the number of clusters; however, it is not always possible to know how many color image segments are within a color image in advance. Therefore, to exploit the clustering task in a full automatic way, the clustering algorithm should contain a procedure to determine the optimal number of clusters from data itself without the user's specification.

To achieve this, the cluster validity index, $v_{o s}$, proposed by Kim et al. [11] is applied to the clustering results obtained through the S-FCM algorithm to validate the quality of the partition. The $v_{o s}$ index searches for the optimal number of clusters using two properties: an overlap measure and a separation measure. A good cluster result is expected to have a low degree of overlap and a large separation distance. We refer to this procedure as a validityguided S-FCM (VS-FCM) algorithm. This procedure is repeated with respect to the possible candidates for the number of clusters. Of the partitions considered, the partition with the minimum value of $v_{o s}$ is identified as the optimal clustering result. Formally given input $X=\left\{x_{1}, \ldots, x_{n}\right\}$, $R=\left\{r_{1}, \ldots, r_{k}\right\}, m, \epsilon$, and $d$, the VS-FCM procedural steps are described as follows:

S1: $c \leftarrow 2, c_{\text {max }} \leftarrow \sqrt{n}$, and $v_{o s}^{\text {min }} \leftarrow 0 ;$
S2: while $c \leq c_{\max }$ do $(\mathrm{S} 3, \mathrm{~S} 4, \mathrm{~S} 5)$
$\mathrm{S} 3: F^{c} \leftarrow$ the partition obtained by the S-FCM

S4: compute $v_{o s}\left(F^{c}\right)=\operatorname{Overlap}\left(F^{c}\right) / \operatorname{Sep}\left(F^{c}\right)$

S5: $c \leftarrow c+1$ and go to S2

S6: Report $F^{c}$ with $\min v_{o s}$ as the optimal partition;

\section{Experiments and Conclusion}

To test the effectiveness of the proposed scheme, the FCM, S-FCM, and VS-FCM were applied to image segmentation problems; the weighing exponent was set to be $m=2.0$ since it have showed the overwhelming favorite [1]; the termination criteria was set to be $\epsilon=0.001$; in FCM, the initial centroids were selected by random; the color data $x_{j} \in X$ in an image were represented in the CIELAB color space due to its superior performance to other spaces [9]; 14 distinguishable colors were chosen from the the Munsell Color system to compose the reference data set [12].

The size of the spatial neighborhood $S_{j}$ has influence on the value of the homogeneity. As Cheng et al. pointed out [10], a small value of $S_{j}$ is limited to reflect local information in the calculation of the homogeneity. In contrary, a large value of $S_{j}$ requires a high computation time. Thus, 
in this study we chose a $3 \times 3$ window for $S_{j}$ by weighing the trade-off.

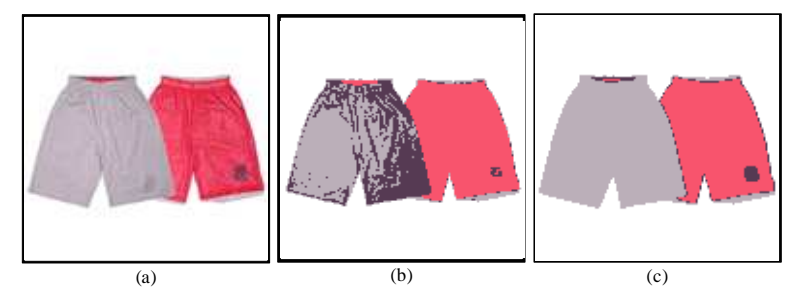

Fig. 1 Comparison of the segmentation results using FCM and S-FCM: (a) original image "pants" (b) FCM (c) S-FCM

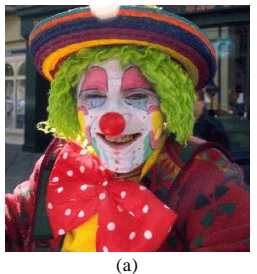

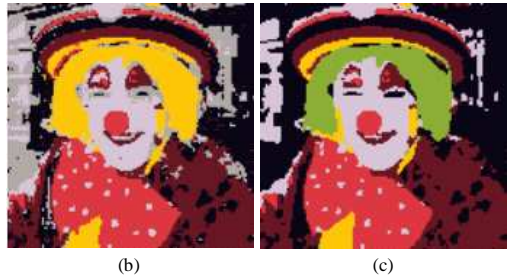

Fig. 2 Comparison of the segmentation results using FCM and S-FCM: (a) original image "clown" (b) FCM (c) S-FCM

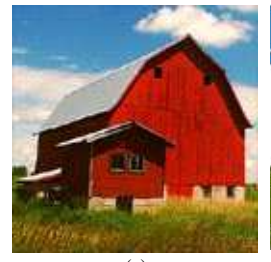

(a)

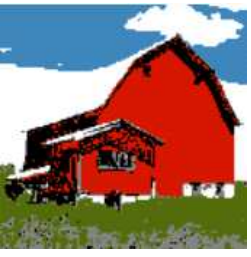

(b)

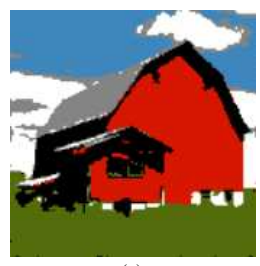

(c)
Fig. 3 Comparison of the segmentation results using FCM and S-FCM: (a) original image "house" (b) FCM (c) S-FCM

Firstly, we compared the clustering performance of the S-FCM with the FCM method. Figure 1(a) shows an original image ("pants") containing four colors $(c=4)$ : a white for the background, a gray for the left-side pants, a red for the right-side pants, and black for the rectangular mark on the red pants. (Please note that this figure must be viewed in color to appreciate the color segmentation results.) An ideal clustering would result in four segmented objects. Many color points on the gray pants are close to those of the rectangular mark on the red pants in the color space. Thus, clustering based on the FCM algorithm [Figure 1(b)] led to an over-segmentation in the left-side (gray) pants due to the large number of creases in those pants, and the black mark is not clear. In contrast, those components were clearly segmented by the S-FCM algorithm [Figure 1(c)]; it prevented the FCM from falling into incorrect local optima.
Figure 2(a) shows an original image ("clown") containing various colors. Each clustering algorithm was applied to segment the image into six colors $(c=6)$ : white, black, red, brown, yellow, and green. The FCM algorithm failed to give correct clustering results [Figure 2(b)]; for example, the green hair was misclassified as yellow color in the final image. In contrast, the S-FCM algorithm [Figure 2(c)] successfully segmented the six colors where the hair was clearly classified since the green color was identified as one of the dominant colors from the image.

Figure 3(a) shows an original image ("house") where each clustering algorithm was applied to segment the image into six clusters $(c=6)$ : white, blue, red, black, gray, and green. The FCM algorithm failed to give a correct clustering result [Figure 3(b)]; the bottom (green) grass was oversegmented and the (gray) roof of the house was misclassified as white color. In contrast, the S-FCM algorithm provided a better segmentation result [Figure 3(c)]; the grass, the roof and the (black) shade were more clearly classified.

Table1. Cluster validity values for the "clown" image for the VS-FCM with respect to $c=2, . ., c_{\max }=7$. The bold face indicates the optimal result for the given validity index.

\begin{tabular}{ccccccc}
\hline$c$ & $v_{P C}$ & $v_{P E}$ & $v_{X B}$ & $v_{K}$ & $v_{S C}$ & $v_{O S}$ \\
\hline 2 & $\mathbf{0 . 5 7}$ & $\mathbf{0 . 2 3}$ & 0.29 & 4743.95 & $\mathbf{- 0 . 7 8}$ & 1.12 \\
3 & 0.54 & 0.29 & $\mathbf{0 . 1 2}$ & $\mathbf{1 9 6 9 . 1 5}$ & -1.40 & 0.58 \\
4 & 0.49 & 0.35 & 0.23 & 3729.60 & -2.10 & 0.49 \\
5 & 0.50 & 0.37 & 0.18 & 3009.77 & -2.63 & 0.39 \\
$\mathbf{6}$ & 0.49 & 0.40 & 0.16 & 2584.05 & -3.10 & $\mathbf{0 . 3 7}$ \\
7 & 0.44 & 0.44 & 0.40 & 6577.64 & -4.28 & 0.41 \\
\hline
\end{tabular}
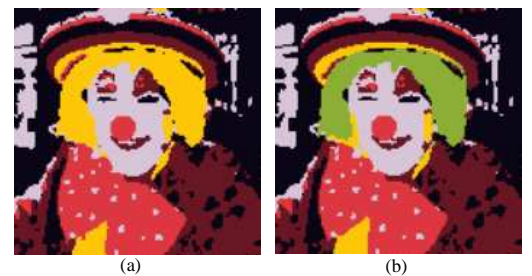

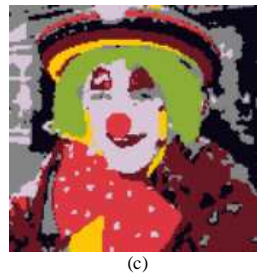

Fig. 4 Comparison of the segmentation results using VS-FCM for different values of $c$ : (a) VS-FCM with $c=5$ (b) VS-FCM with $c=6$ (c) VS-FCM with at $c=7$

Secondly, to show the potential of the VS-FCM, we tested several comparisons with other validity indexes for $c=$ $2, \ldots, c_{\max }$. The detailed explanation of each validity index can be found in [11]. For the "clown" image [Figure 2(a)], we find that the VS-FCM using $c=6$ generates the optimal clustering result [Table 1]; only $v_{\text {os }}$ correctly recognizes $c=6$ whereas all other indexes point to $c=2$ or $c=3$ as the optimal partition. Figure 4 shows three of the six clustering results for the VS-FCM for different values of $c$; From the figure, we find that VS-FCM with $c=6$, 
in figure 4(b), shows better result than the other two cases of VS-FCM with $c=5$ [fig. 4(a)] and VS-FCM with $c=7$ [fig. 4(c)].

From the experiments, we see that the results presented above highlight the potential of the proposed approach to image segmentation; the VS-FCM makes it possible for user not to explicitly specify the number of clusters a priori. Acknowledgement. This work was supported by Basic Science Research Program through the National Research Foundation of Korea(NRF) grant funded by the Korea government(MEST)(No.2009-0064861)

\section{References}

[1] J. C. Bezdek et al, Fuzzy Models and Algorithms for Pattern Recognition and Image Processing, Kluwer Academic Publishers, Boston, 1999.

[2] A. K. Jain, M. N. Murty, P. J. Flynn, "Data clustering: a review," ACM Computing Surveys, vol. 31, pp. 264-323, 1999.

[3] N. S. Iyer, A. Kandel, M. Schneider, "Feature-based fuzzy classification for interpretation of mammograms," Fuzzy Sets and Systems, vol. 114, pp. 271$280,2000$.

[4] A. Shahin, M. Menard, M. Eboueya, "Cooperation of fuzzy segmentation operators for correction aliasing phenomenon in 3D color doppler imaging," Artificial Intelligence, vol. 19, pp. 121-154, 2000.

[5] Y. A. Tolias, S. M. Panas, "Image segmentation by a fuzzy clustering algorithm using adaptive spatially constrained membership functions," IEEE Trans. Syst. Man Cybern. A, Syst. Humans, vol. 28, pp. 359369, 1998.

[6] A. W. C. Liew, S. H. Leung, W. H. Lau, "Fuzzy image clustering incorporating spatial continuity," IEE Proceedings of Vis. Image Process, vol. 147, pp. 185$192,2000$.

[7] D. Qzdemir, L. Akarun, "A fuzzy algorithm for color quantization of images," Pattern Recognition, vol. 35, pp. 1785-1791, 2002.
[8] D. W. Kim, K. H. Lee, D. Lee, "A novel initialization scheme for the fuzzy c-means algorithm for color clustering," Pattern Recognition Letters, vol. 25, pp. 227-237, 2004.

[9] G. Paschos, "Perceptually uniform color spaces for color texture and analysis," IEEE Transactions on Image Processing, vol. 10, pp. 932-937, 2001.

[10] H. -D. Cheng, Y. Sun, "A hierarchical approach to color image segmentation using homogeneity," IEEE Transactions on Image Processing, vol. 9, pp. 20712082, 2000.

[11] D. W. Kim, K. H. Lee, D. Lee, "On cluster validity index for estimation of the optimal number of fuzzy clusters," Pattern Recognition, vol. 37, pp. 20092025, 2004.

[12] J. T. Luke, The Munsell color system: a language for color, Fairchild Publications, New York, 1996.

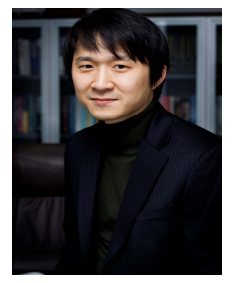

\section{Dae-Won Kim}

He received the M.S. and Ph.D degrees in computer science from Korea Advanced Institute of Science and Technology (KAIST), Daejeon, Korea, in 1999 and 2004. Since 2005, he has been an assistant professor at the School of Computer Science and Engineering, Chung-Ang University, Korea. His research interests include data mining, pattern recognition, and artificial intelligence.

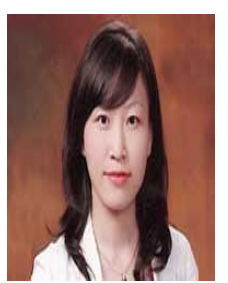

\section{Bo-Yeong Kang}

She received the M.S. and Ph.D degrees in computer engineering from Kyungpook National

University, Daegu, Korea, in 2002 and 2004, respectively. She is currently an assistant professor at the School of Mechanical

Engineering, Kyungpook National University, Korea. Her current research interests include machine intelligence technology, robotics, and biomedical system. 